\title{
Morphology of Extruded High-Density Polyethylene Pipes Studied by Atomic Force Microscopy
}

\author{
DORINA TRIFONOVA, ${ }^{1}$ PHILIPPE DROUILLON, ${ }^{2}$ ANTOINE GHANEM, ${ }^{2}$ G. JULIUS VANCSO ${ }^{1}$ \\ ${ }^{1}$ University of Twente, Faculty of Chemical Technology, P.O. Box 217, NL-7500 AE Enschede, The Netherlands \\ ${ }^{2}$ SOLVAY S.A. Direction Centrale des Recherches, Laboratoire Central, rue de Ransbeek 310, B-1120 Bruxelles, Belgium
}

Received 30 December 1996; accepted 7 April 1997

\begin{abstract}
Atomic force microscopy (AFM) was used to study the structure of extruded polyethylene (PE) pipe. During extrusion, the outer surface of the pipe was cooled with water. Two cross sections, parallel and transverse to the extrusion direction, were examined in order to spatially follow the structural development during extrusion. The morphology revealed was spherulitic, and the spherulites had a mostly banded appearance when viewed under the AFM. We were not able to distinguish an oriented skin layer at the surface of the pipe, either by AFM or polarizing microscopy. The changes in the pipe's structure resulting from the cooling conditions were found to be rather gradual, and no clearly defined zones were observed. A slight orientation towards the extrusion direction was detected only in the area of the pipe crystallized under the lowest degree of undercooling. Measured spherulitic size, band period, and lamellae thickness showed a gradual increase in their values from the cooled to the noncooled surface of the pipe. Transmission electron microscopy (TEM) was used to verify the band period and lamellae thickness measurements done by AFM. (C) 1997 John Wiley \& Sons, Inc. J Appl Polym Sci 66: 515-523, 1997
\end{abstract}

Key words: atomic force microscopy; polyethylene; pipe extrusion; morphology; banded spherulites

\section{INTRODUCTION}

Polyethylene (PE) is one of the most widely used polymers because it is inexpensive and has excellent physical properties. The different properties of PE depend, to a great extent, upon the morphologies formed as a result of processing conditions, heat treatment, as well as upon molecular parameters such as branching, molecular weight, and molecular weight distribution. ${ }^{1}$

Fabrication-controlled structural differences can result in variations in orders of magnitude of the mechanical characteristics of $\mathrm{PE}^{2,3}$ Thus, it is important to better understand the structures formed during processing and the conditions that

\footnotetext{
Correspondence to: G. Julius Vancso.

Journal of Applied Polymer Science, Vol. 66, 515-523 (1997)

(C) 1997 John Wiley \& Sons, Inc.

CCC 0021-8995/97/030515-09
}

cause their formation. One of the important aspects of this understanding concerns gaining a detailed knowledge of the associated morphology.

For a standard production process like extrusion, most of the morphological studies on PE products have concentrated on high modulus materials showing highly oriented structures such as fibrils, shish-kebabs, etc. ${ }^{4-11}$ These materials were usually produced under special conditions in order to achieve high degrees of orientation. Very little has been published about the structures formed in conventionally extruded PE products.

Skin-core morphology is usually observed in injection-moulded plastics. ${ }^{12,13}$ For example, Tan and Kamal ${ }^{13}$ extensively studied the skin-core morphology of injection-moulded PE. They observed four distinct zones in the cross section. Zone 1 was nonspherulitic and exhibited row-like features growing in the depth direction. According 
to the authors, these rows could be interpreted as stacks of lamellae with chain axes oriented in the flow direction. Zone 2 consisted of very fine asymmetric spherulites of an almost uniform size, zone 3 showed oblate spherulites with axis of symmetry in the direction of the thermal gradient, and zone 4 displayed randomly nucleated spherulites, which grew in all directions until impingement. A similar structure, consisting of an oriented skin and a spherulitic core, was seen for extruded pellets of polyoxymethylene. ${ }^{2}$ Thus, intuitively, one might expect that extruded PE pipes would also exhibit skin-core morphologies. Terselius et al., ${ }^{14}$ on the other hand, reported a nondifferentiated spherulitic morphology in both the bulk and the inner surface of extruded PE pipes. However, these pipes were cooled from the outside, and the authors did not discuss the structure of the external surfaces. Thus, the overall picture of the morphology of PE pipes has not yet been conclusively given.

Most of the knowledge about the structures developed in extruded and injection-moulded plastics has come from optical and scanning electron microscopy ( SEM) images. In recent years, atomic force microscopy (AFM) has been established as a powerful tool for investigating polymers. ${ }^{15}$ This technique has shown great potential for imaging polymeric surfaces over a wide range, from microto nanometric scales. ${ }^{16}$ Fine details of spherulitic and lamellar morphologies have been visualized for a variety of polymers. ${ }^{17,18}$ Many studies have focused on nanoscale structures of highly oriented systems. $^{19,20}$

In this article, we present the results of an AFM investigation of the structures formed in extruded PE pipe. Optical microscopy and transmission electron microscopy (TEM) were also used in this study: the first to give a general overview of the investigated structures; the latter to compare TEM results with AFM images. Two cross sections, parallel and transverse to the extrusion, were examined in order to spatially elucidate the structural development during extrusion.

\section{EXPERIMENTAL}

The specimens were taken from part of an extruded pipe, which was made of a non-pigmented, commercial, high-density PE resin. The polymer had a weight-average molar mass of $2.5 \times 10^{4} \mathrm{~g}$ $\mathrm{mol}^{-1}$, and a density of $949 \mathrm{~kg} \mathrm{~m}^{-3}$. The processing was conventional, i.e., melt extrusion (at $197^{\circ} \mathrm{C}$ ) through an annular die at a rate of $265 \mathrm{~cm} \mathrm{~min}^{-1}$, followed by calibration and water cooling $\left(27^{\circ} \mathrm{C}\right)$ of the outer surface. The thickness and the external diameter of the pipe were about 3 and $50 \mathrm{~mm}$, respectively. For convenience, we introduced cylindrical coordinates $(z, r, \varphi)$ to describe the locations of the specimens cut from the pipe. The extrusion (machine) direction was $z$, and the pipe thickness varied between $r=22 \mathrm{~mm}$ and $r=25$ $\mathrm{mm}$. The specimen that exhibited a representative $(z, r)$ plane at $\varphi=$ constant is referred to as a parallel cross section, while the specimen that showed a typical $(r, \varphi)$ plane at $z=$ constant is referred to as a transverse cross section. We assume that the morphology has a cylindrical symmetry, i.e., that the morphology does not depend on $\varphi$ for a given $r$ and $z$.

For AFM imaging, specimens were cut from the cross section of the pipe, smoothened by ultramicrotoming at $-90^{\circ} \mathrm{C}$, and then etched at room temperature. The composition of the etchant was $0.7 \% \mathrm{w} / v$ of potassium permanganate dissolved in a mixture of two volumes of concentrated sulphuric acid and one volume of orthophosphoric acid. After etching, the specimens were washed according to published procedures ${ }^{21,22}$ and were dried prior to examination.

Specimens for TEM were taken from about the middle part of the transverse cross section of the pipe, i.e., from the $(z, \varphi)$ plane at ca. $r=23.5 \mathrm{~mm}$ radius. The lamellar organization was revealed after soaking the specimens (which had a trimmed pyramidal shape) in a mixture of ruthenium trichloride and aqueous sodium hypochloride. ${ }^{23}$ Sections of $90 \mathrm{~nm}$ nominal thickness, perpendicular to the extrusion direction, were cut at $-95^{\circ} \mathrm{C}$ using a diamond knife and a Reichert Ultracut S ultramicrotome. Specimens were subsequently collected on standard copper grids and were examined with a Siemens Elmiskop 102 electron microscope at $80 \mathrm{kV}$. Sections $15 \mu \mathrm{m}$ thick were taken in the machine and transverse directions for tests with polarized optical microscopy.

The AFM measurements were carried out with a NanoScope ${ }^{\circledR}$ III set up (Digital Instruments, Santa Barbara, CA). The instrument was equipped with $\mathrm{J}$ and $\mathrm{D}$ scan heads and was calibrated in the $x$ and $y$ directions by using the images of a calibration grid and gold calibration grating. All scans were performed in air with $\mathrm{Si}_{3} \mathrm{~N}_{4}$ NanoProbe integrated microcantilevers with a nominal force constant of $0.38 \mathrm{~N} \mathrm{~m}^{-1}$ (NanoTips ${ }^{\circledR}$, Digital Instruments ). NanoProbes used to perform the scans were carefully selected after imaging the (305) surface of a $\mathrm{SiTiO}_{3}$ crystal following the procedure described by Sheiko et al. ${ }^{24}$ 
The scan rates selected were between 1.8 and 8 $\mathrm{Hz}$, depending on the scan size. Height ( $\mathrm{HM}$ ) and deflection (DM) images were registered simultaneously. DM could not be used for quantitative measurements of the topology but proved to be very useful for observation of the surface features. All AFM micrographs shown in this article correspond to height images. Imaging forces were minimized to $20 \mathrm{nN}$ in order to obtain the best contrast. It should be pointed out that the structures observed remained essentially the same in scans with different forces ranging between 20 and $30 \mathrm{nN}$.

\section{RESULTS AND DISCUSSION}

Structures that developed during extrusion are shown in Figures 1 and 2. The AFM micrographs were chosen to show typical images of the scanned areas of the specimens. Scans were performed on specimens in both the $(z, r)$ and $(r, \varphi)$ planes. A large number of scans were captured. Near the cooled edge, where one might expect to have a skin structure, the specimens were imaged in 0.05 mm steps between $r=25 \mathrm{~mm}$ and $r=24.7 \mathrm{~mm}$. For the rest of the specimen, scans were performed every $0.4 \mathrm{~mm}$, until the noncooled edge was reached. In Figure 1(a)-(e), typical AFM scans obtained in the $(r, \varphi)$ plane (transverse direction) are given. Figure 2(a) and (b) show a comparison of the morphology in the transverse and parallel directions, captured at a higher magnification. The overall morphology of the specimens, as one can see in Figures 1(a) - (e), shows a spherulitic appearance. We did not observe oriented skin structures near the surface of the specimen. This might be due to the etching procedure applied to the specimens. During this procedure, the surface of the specimen is oxidized, causing a rounding of sharp features such as edges. A very thin skin layer might be removed by the etchant. However, when viewed under a polarizing microscope between crossed polars, unetched specimens did not show bright stripes, corresponding to a birefringent skin, running parallel to the surface. Such features are usually seen in specimens made by injection moulding, and are commonly referred to as the skin. ${ }^{2,3}$ One explanation for the absence of the skin layer is the fact that its nature and depth are related to the critical flow and thermal phenomena occurring during the injectionmoulding process. ${ }^{3}$ In addition to these factors, the formation of such a layer can also be influenced by the contact of the melt with the mould wall. ${ }^{3,14}$ In the case of extrusion, the main processing parameters influencing the structure of extruded pipes are the flow rate, the temperature of the melt, and the cooling conditions.

The spherulites observed in the AFM height images have a banded appearance throughout most of the specimen. Under the polarizing microscope, however, this banding is almost unrecognizable. This could be explained by the fact that the spherulitic diameter and band spacing were often smaller than the specimen thickness. Therefore, most of the images obtained in transmitted polarized light were the result of superimposed images of different spherulites; this would have obscured the banded appearance. It should be pointed out that there are different reasons for this appearance in the two imaging techniques that were used. Under the polarizing microscope, the dark bands are periodic birefringent extinction patterns caused by a regularly twisted molecular orientation about the radial growth axis. In the AFM images on the other hand, differences in the gray scale represent different heights of the surface features, with brighter tones corresponding to higher regions and darker tones corresponding to lower regions. We will later show that the origin of the periodic difference in height seen in the AFM images is the same as for the birefringent bands.

It was mentioned above that in extruded and injection-moulded specimens, one can normally observe zones with different morphologies that result from the processing conditions. ${ }^{3}$ In our study, however, the change in the morphology from the cooled to the noncooled edge of the pipe was rather gradual, and we were not able to distinguish between clearly defined zones. One can follow changes in the structure along the two investigated cross sections. Apart from some deformation of the spherulites in the machine direction (which can be seen on images of the parallel cross sections near the noncooled edge), the features shown in the $(r, z)$ and $(r, \varphi)$ planes are similar. Thus, we will discuss in detail the images captured in the transverse plane [Fig. 1(a)-(e)]. Near the cooled surface (see Table I), small and mostly asymmetric spherulites are seen [Fig. 1(a)]. This part of the specimen crystallized under the highest undercooling, which determines the high nucleation density. Because the lateral growth of the spherulites is restricted due to their neighbors, they grow asymmetrically and mainly in one direction (in the depth of the specimen from high to lower radii), forming two or three bands. The spherulites farther from the cooled surface, 


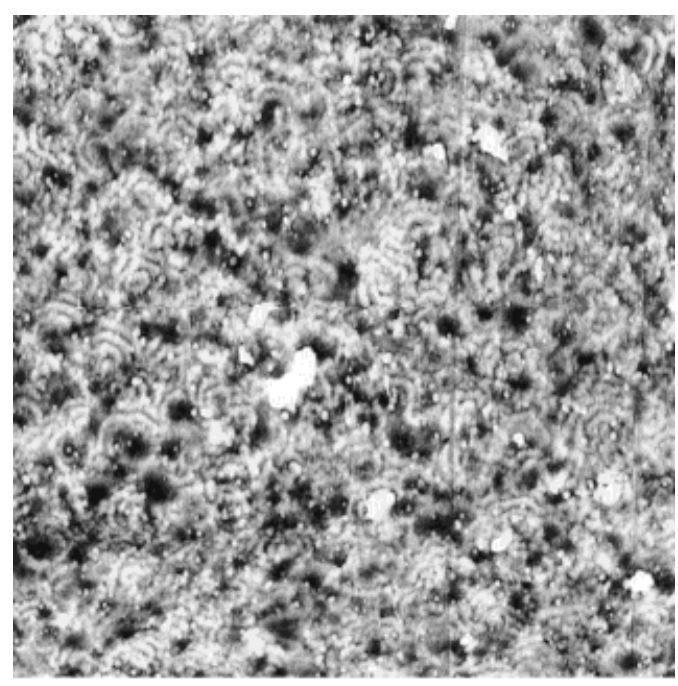

(a)

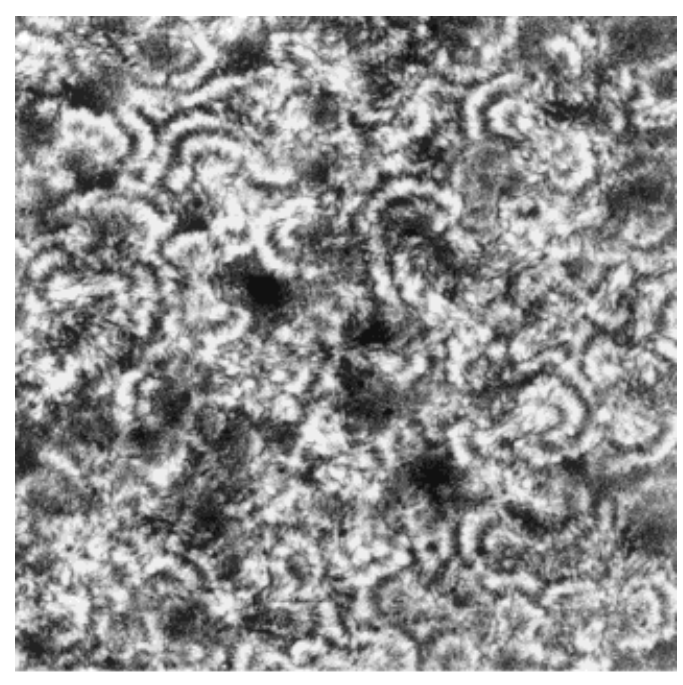

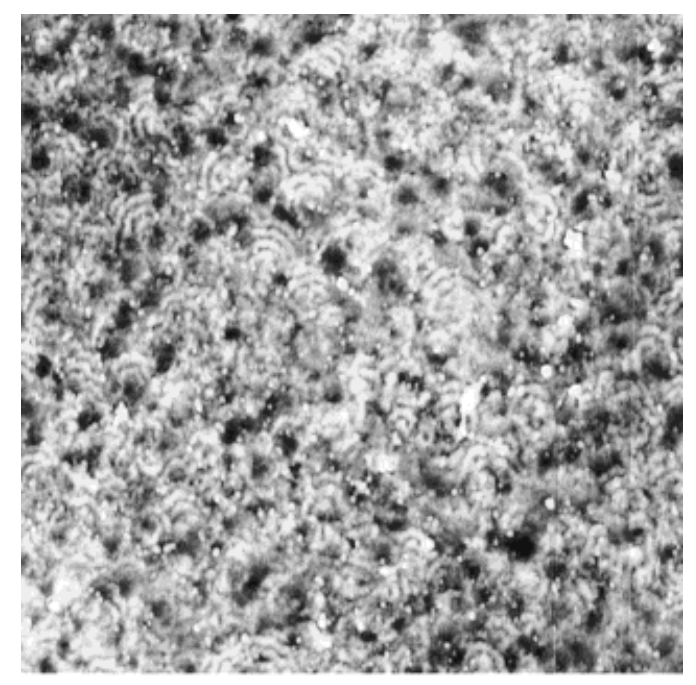

(b)

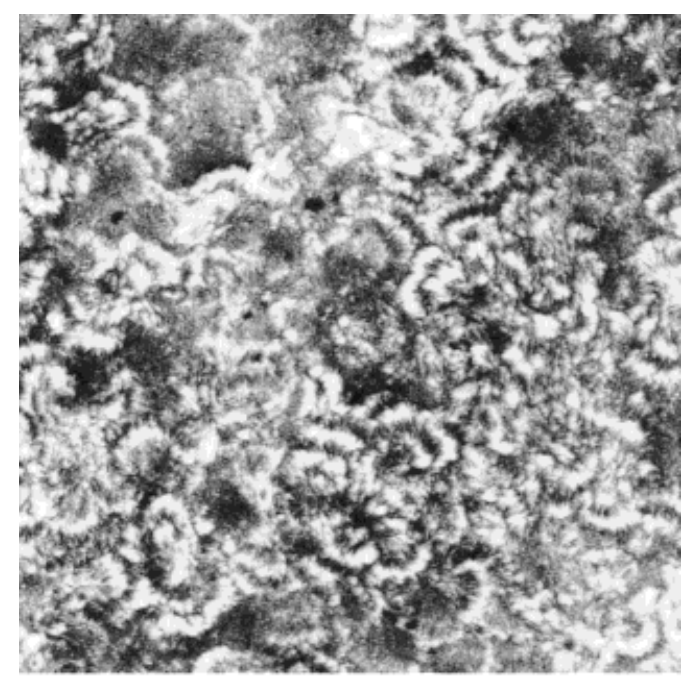

(c)

(d)

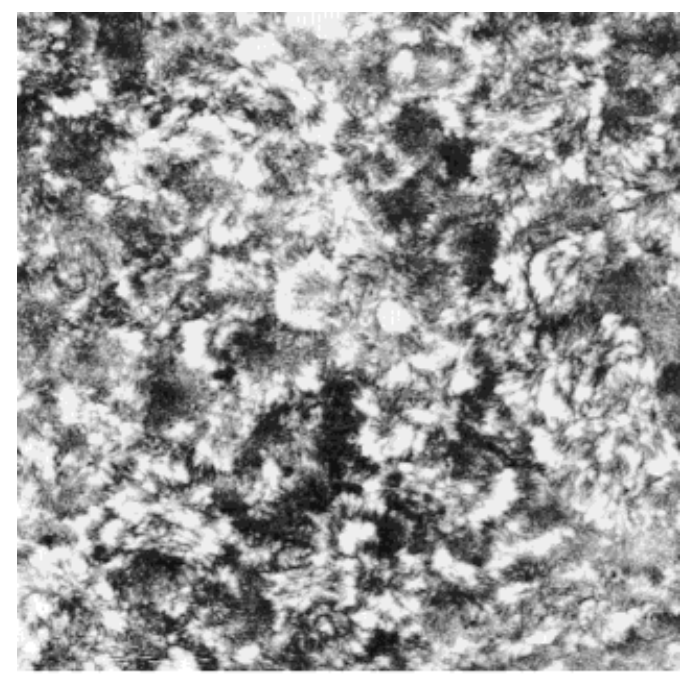

(e) 

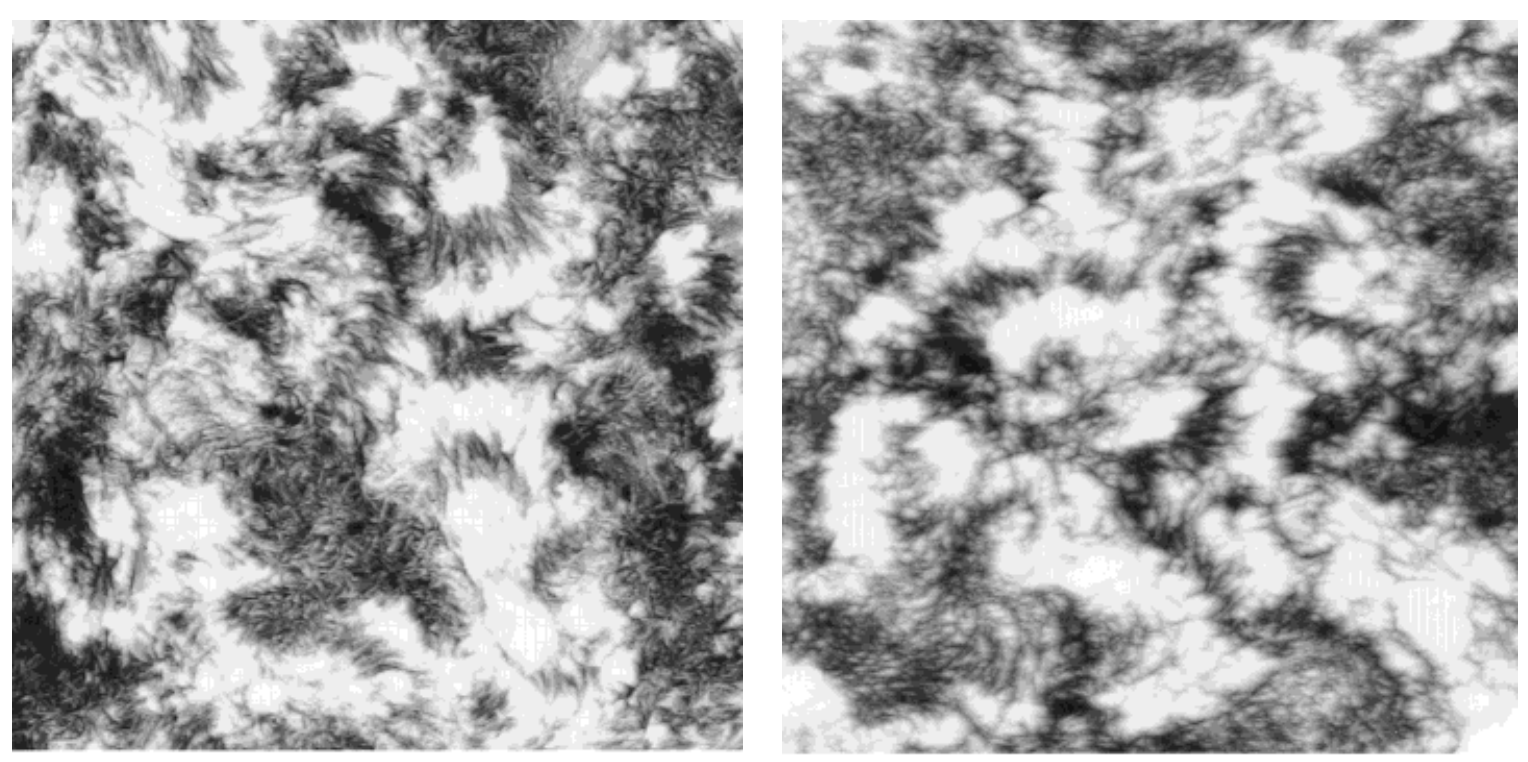

(a)

(b)

Figure 2 AFM scans showing the morphology near the inside surface of the pipe (noncooled edge). Scan size: $15 \times 15 \mu \mathrm{m}$. Gray scale for the feature heights: black $=0.0 \mathrm{~nm}$; white $=880 \mathrm{~nm}$. (a) Parallel cross section, in which the cooling direction is from the right to the left. (b) Transverse cross section, in which the cooling direction is from the bottom to the top.

at smaller values of $r$, become larger and more rounded [Fig. 1(b)]. The band period is bigger as well and varies between 800 and $900 \mathrm{~nm}$ at this part of the specimen. Deeper into the specimen, most of the spherulites become rounder, and almost no asymmetrical growth can be seen. Apparently, at this radius (about $23.5 \mathrm{~mm}$ ), the thermal gradient is low, and the spherulites are randomly nucleated, growing until they impinge upon each other. Moving towards the inner surface that was not cooled, both the size and band period of the spherulites gradually increase, reaching the values of 13 and $2.0 \mu \mathrm{m}$, respectively (for a representative image, see Fig. 2). The band period indicated on the TEM image (Fig. 3), taken approximately in the middle of the transverse cross section, was in good agreement with the average band period measured from the AFM images for this area of the specimen.

As mentioned earlier, when comparing the transverse and parallel cross sections, almost no difference in structure can be seen. However, a certain orientation can be seen in the parallel section in the regions close to the noncooled surface (compare Fig. 2(a) with (b)). In contrast to the transverse cross section [in which the spherulites look more or less round, see Fig. 2(b)], in the parallel cross-section, the spherulites have irregular oblate shapes and are oriented in the flow direction [see Fig. 2(a)]. It should be noted as well that in both of the cross sections that were examined, the banded appearance becomes less obvious close to the noncooled surface (Figs. 1(e) and 2), although it is still present. This might be due to the bigger spherulitic size, which causes the less diametrical cross sections of the spherulites to be exposed to the microtomed surface. In the nondiametrical sections, the banded appearance could be obscured, depending on the distance of the cutting plane from the spherulitic centre, and also depending on the angle between the cutting plane and the spherulitic radius.

We expect that the degree of crystallinity will increase towards the noncooled surface because

Figure 1 AFM height images of the cross section transverse to the extrusion direction: (a) cooled edge; (b) midway between the cooled edge and the middle; (c) middle; (d) midway between the middle and noncooled edge; (e) noncooled edge. In all images, the cooling direction is from the bottom to the top. Scan size: $40 \times 40 \mu \mathrm{m}$. Gray scale for the feature heights: black $=0.0 \mathrm{~nm}$; white $=1.20 \mu \mathrm{m}$. 
Table I Estimated Range of Spherulitic Size and Band Period in the Pipe's Cross Section Parallel to the Extrusion Direction ${ }^{a}$

\begin{tabular}{lcc}
\hline \multicolumn{1}{c}{ Imaged Area of the Cross Section } & $\begin{array}{c}\text { Spherulitic Size } \\
(\mu \mathrm{m})\end{array}$ & Band Period \\
\hline Cooled edge & $2-3$ & $400-500 \mathrm{~nm}$ \\
Midway between the cooled edge and the middle & $4-5$ & $800-900 \mathrm{~nm}$ \\
Middle & $6-8$ & $1.0-1.1 \mu \mathrm{m}$ \\
Midway between the middle and the cooled edge & $8.5-10.5$ & $1.2-1.4 \mu \mathrm{m}$ \\
Noncooled edge & $10-13$ & $1.6-2.0 \mu \mathrm{m}$ \\
\hline
\end{tabular}

a The figures shown apply for the transverse cross section as well.

there, the polymer chains will have more time to arrange themselves in well-organized crystals. The overall change in the morphology of the specimen in the parallel and transverse cross sections described above suggests a higher degree of crystallinity at the noncooled surface than at the cooled one. The change in the lamellae thickness, measured from the AFM images (Fig. 4) supports this suggestion. Measurements of the lamellar thickness were made on cross sections of the AFM images. Typical results for both flat-on and edgeon orientation of the lamellae with respect to the specimen surface are shown in Figure 4 . The average value of the lamellae thickness was found to be $16 \mathrm{~nm}$ in the middle of the specimen, increasing to $23 \mathrm{~nm}$ at the noncooled surface. We could not resolve individual lamellae in the area close to the cooled surface. It should be mentioned that in AFM measurements, the lateral dimensions of the lamellae can be sometimes overestimated, due to the convolution of the tip shape with the surface features. ${ }^{17,18,25}$ TEM data (see Fig. 5) yielded an average lamellar thickness of $15 \mathrm{~nm}$ in the middle of the transverse cross-section, which is in fair agreement with the corresponding AFM results.

It was possible to image the lamellae because the permanganic etching applied to the specimen exposed the features of the crystalline morphol-

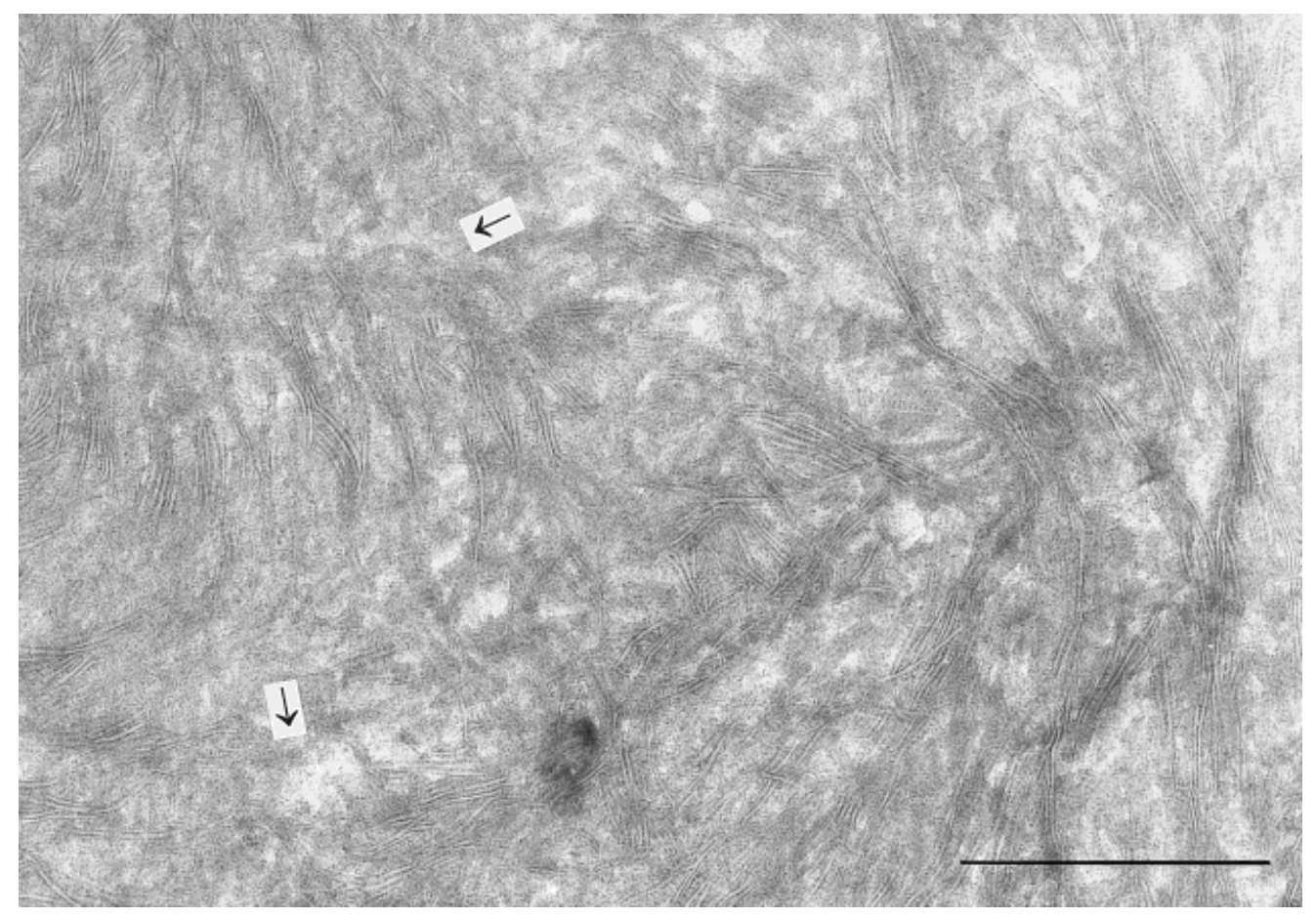

Figure 3 TEM micrographs of a part of a spherulite located in the middle of the cross section transverse to the extrusion direction. Scale bar is $1 \mu \mathrm{m}$. Notice the band period, as indicated by the arrows. 

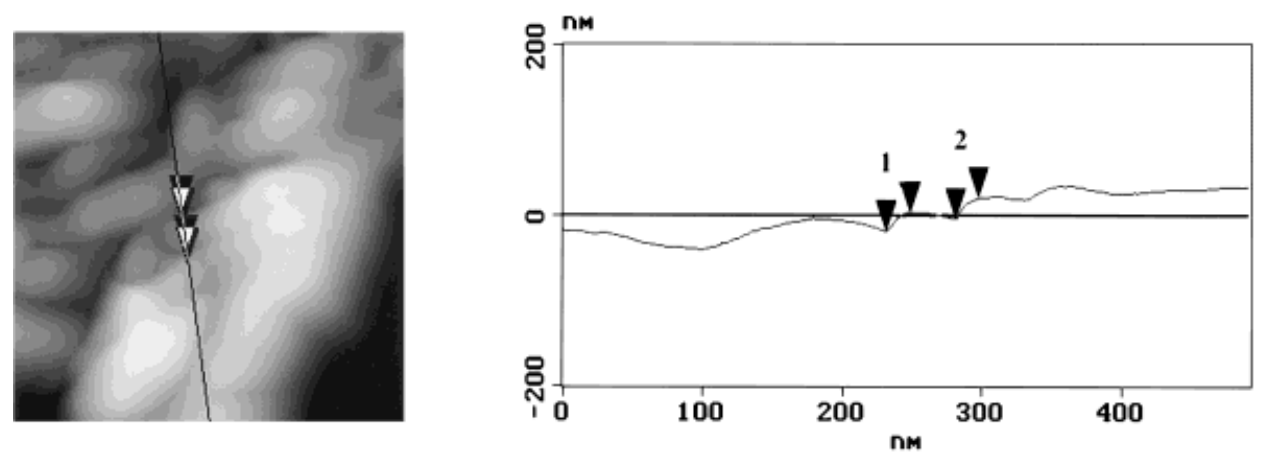

(a)
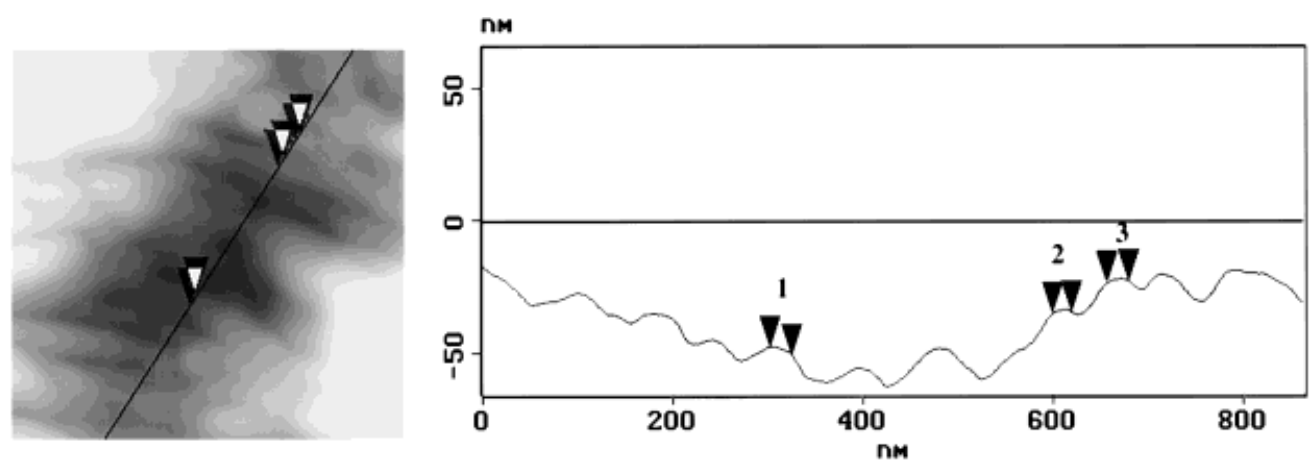

(b)

Figure 4 Section analysis of AFM images showing lamellae thickness in an area of the cross section transverse to the extrusion direction, near to the noncooled surface. (a) Flat-on lamellae. (b) Edge-on lamellae. Estimated lamellar thickness values for the pairs of markers shown: (a) Marker 1, $21.8 \mathrm{~nm}$; marker 2, $23.8 \mathrm{~nm}$. (b) Marker 1, $22.8 \mathrm{~nm}$; marker 2, $20.0 \mathrm{~nm}$; marker 3, $22.9 \mathrm{~nm}$.

ogy. This is a technique that is highly selective in its removal of material from the surface of polymers. It leaves the crystalline lamellae protruding out of an etched surface because it strongly erodes the interlamellar amorphous regions. ${ }^{26}$ The banded appearance of the spherulites observed on the AFM images was caused by different rates of etching due to variations in the orientation of the lamellae at the microtomed surface. The side ('prism' face) surfaces of the lamellae are more easily attacked by the etchant than their basal (fold) surfaces..$^{26,27}$ Therefore, after etching, the basal lamellae surfaces exposed at the microtomed specimen surface were higher (consequently seen in bright tones in the AFM images), and the exposed side lamellae surfaces were lower (seen in dark tones). Thus, the etching of a banded spherulite, where the lamellar orientation is regularly twisted along the radius, produces a systematically undulating topography, which can be clearly seen on surface plots of the spherulites (Fig. 6). This is opposite to the appearance of banded spherulites when using linearly polarized light and crossed polars. In this case, the basal surfaces of the lamellae oriented parallel to the specimen surface cause an extinction pattern and are consequently dark because the molecular chains are parallel to the beam direction, while the side lamellae surfaces are bright because the molecular chains are at an angle to the beam direction.

\section{SUMMARY AND CONCLUSIONS}

In this work, the AFM technique was used to study the morphologies formed in an extruded PE pipe. The structure revealed was entirely spherulitic, showing a banded appearance. The formation of the structure was influenced mainly by the cooling conditions. No oriented skin layer was detected. Along the two investigated cross sections, parallel and transverse to the extrusion direction, a gradual change in the morphology was 


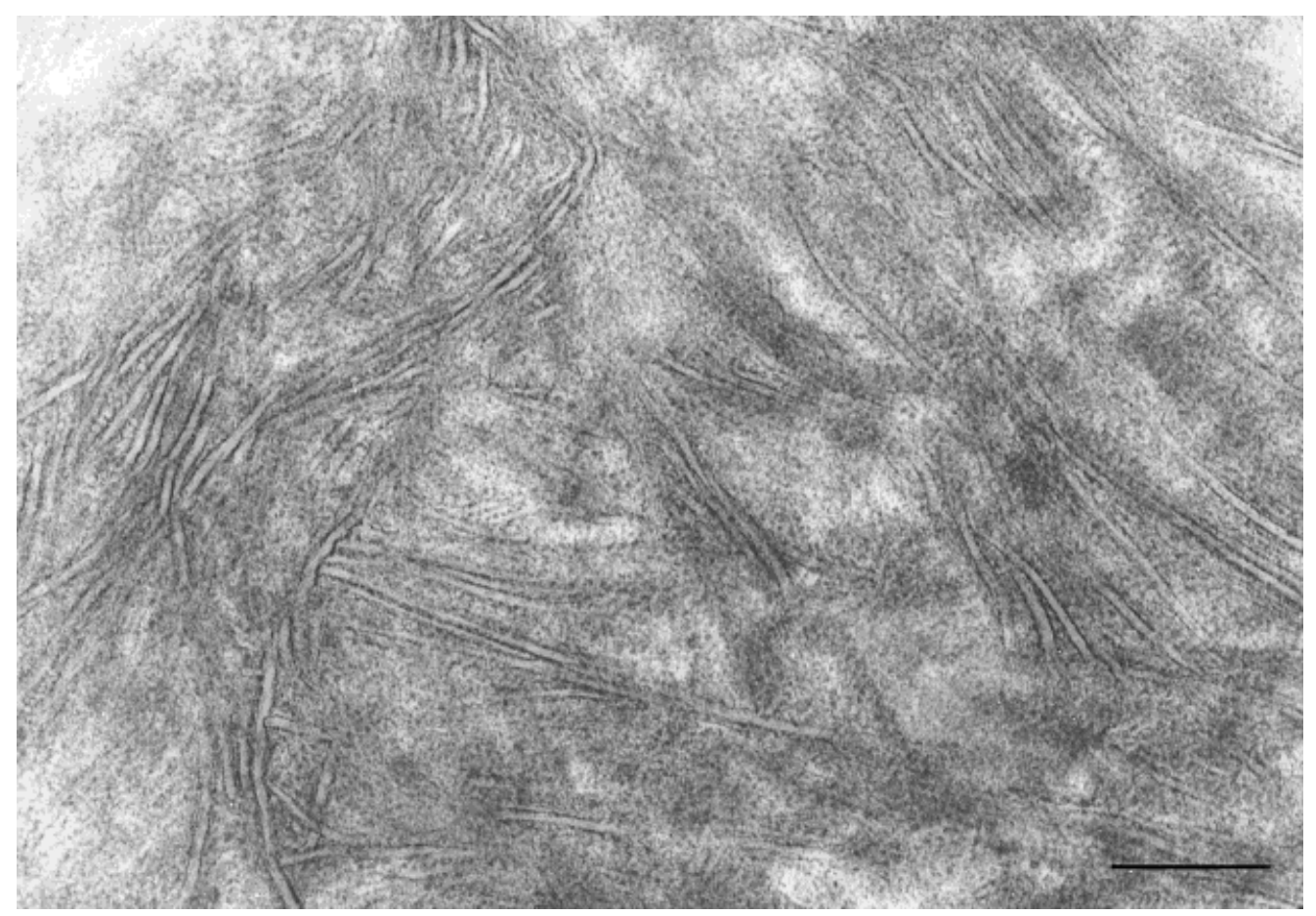

Figure 5 TEM micrographs of a part of a spherulite located in the middle of the cross section transverse to the extrusion direction. Scale bar is $200 \mathrm{~nm}$. Notice the lamella thickness.

observed from small asymmetric spherulites at the cooled edge to randomly nucleated roundshaped spherulites in the interior of the pipe wall. The difference in the structure of the two cross sections was slight; a certain orientation in the extrusion direction was only noticeable in the area of the parallel cross section crystallized under the lowest undercooling. The observed morphology

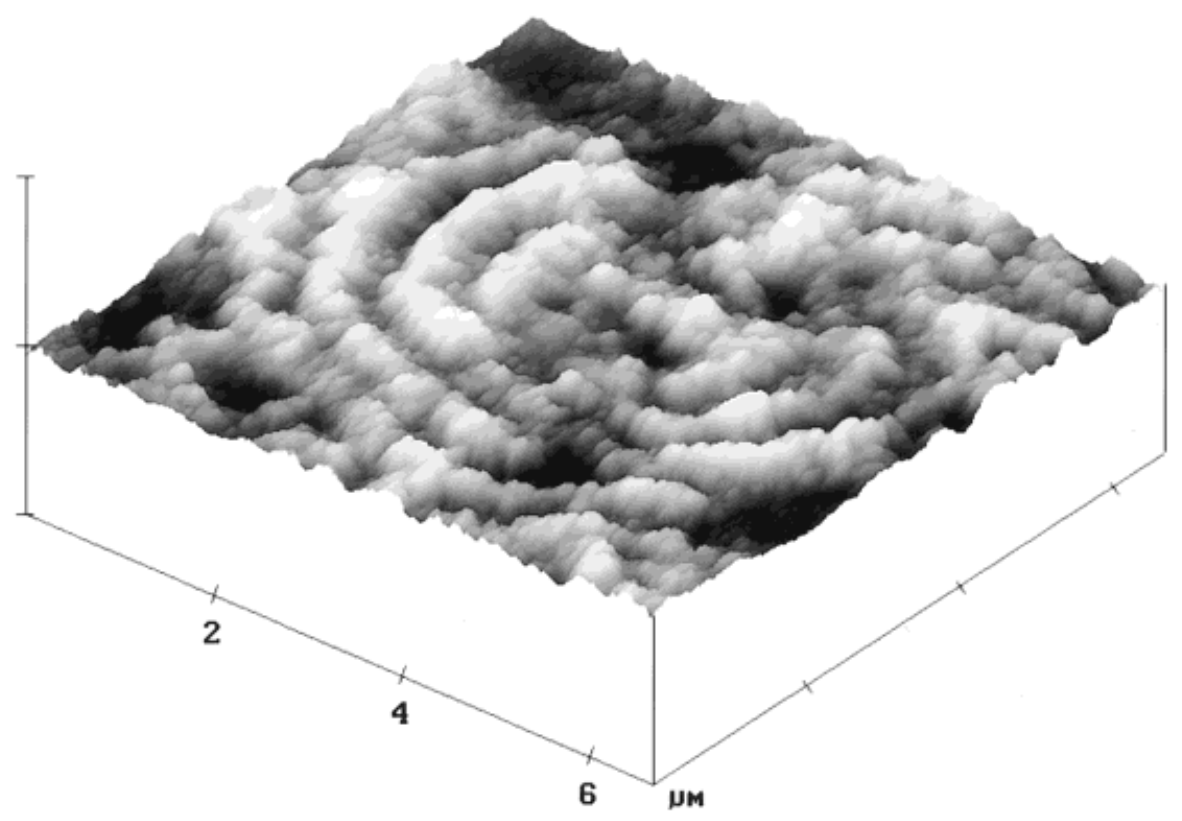

Figure 6 AFM surface plot of a spherulite in the middle of the sample showing the topography resulting from etching. Transverse cross section. Vertical scale is $0.9 \mu \mathrm{m}$ div. 
implies that the mechanical properties both along and across the pipe do not differ very much. These findings contrast with the results of studies on highly oriented extrusion products, which have strongly anisotropic properties.

The authors thank SOLVAY for allowing the publication of these results and Ms. H. Drèze for helpful discussions. D.T. and G.J.V. acknowledge financial support given by the University of Twente. The authors thank Ms. Anne Klemperer for her help with the editing of the manuscript and Mr. R. Flahaux for his assistance with the electron microscopy work.

\section{REFERENCES}

1. J. A. Brydson, in Plastic Materials, 6th ed., Butterworth-Heinemann, Oxford, 1995, Chap. 10.

2. L. C. Sawyer and D. T. Grubb, in Polymer Microscopy, 2nd ed., Chapman \& Hall, London, 1996, Chap. 5.

3. S. S. Katti and J. M. Schultz, Polym. Eng. Sci., 22, 1001 (1982).

4. J. H. Southern and R. S. Porter, J. Macromol. Sci., Phys., B4, 541 (1970).

5. R. G. Crystal and J. H. Southern, J. Polym. Sci., Part A-2, 9, 1641 (1971).

6. J. A. Odell, D. T. Grubb, and A. Keller, Polymer, 19, 617 (1978).

7. E. S. Sherman, R. S. Porter, and E. L. Thomas, Polymer, 23, 1069 (1982).

8. G. Rizzo, S. Piccarolo, and G. Titomanlio, Polym. Commun., 24, 174 (1983).
9. J. A. Odell, A. Keller, and M. J. Miles, Coll. Polym. Sci., 262, 683 (1984).

10. Z. Bashir, J. A. Odell, and A. Keller, J. Mater. Sci., 21, 3993 ( 1986 ).

11. H. T. Yu and G. L. Wilkes, Polym. Prepr., 35, 785 (1994).

12. M. R. Kantz, H. D. Newman, and F. H. Stigale, J. Appl. Polym. Sci., 16, 1249 (1972).

13. V. Tan and M. R. Kamal, J. Appl. Polym. Sci., 22, 2341 (1978).

14. B. Terselius, U. W. Gedde, and J.-F. Jansson, Polym. Eng. Sci., 22, 422 (1982).

15. S. N. Magonov and M. H. Wangbo, Surface Analysis with STM and AFM, VHC, Weinheim, 1996.

16. M. J. Miles, in Characterization of Solid Polymers, S. J. Spells, Ed., Chapman \& Hall, New York, 1994, Chap. 2.

17. H. Schönherr, D. Snetivy, and G. J. Vancso, Polym. Bull., 30, 1567 (1993).

18. G. J. Vancso, R. Nisman, D. Snetivy, H. Schönherr, P. Smith, C. Ng, and H. Yang, Colloids \& Surfaces A, 87, 263 (1994).

19. D. Snetivy and G. J. Vancso, Colloids \& Surfaces A, 87, 257 (1994).

20. G. J. Vancso, Polym. Prepr., 37(2), 550 (1996).

21. R. H. Olley, A. M. Hodge, and D. C. Bassett, J. Polym. Sci., Polym. Phys. Ed., 17, 627 (1979).

22. R. H. Olley and D. C. Bassett, Polymer, 23, 1707 (1982).

23. D. Montezinos, E. Gail Wells, and J. L. Burns, J. Polym. Sci., Polym. Phys. Ed., 23, 421 (1985).

24. S. S. Sheiko, M. Möller, E. M. C. M. Keuvekamp, and H. W. Zandbergen, Phys. Rev., Part B, 48, 5675 (1993).

25. P. Markiewicz and M. C. Goh, Langmuir, 10, 5 (1994).

26. R. H. Olley, Sci. Prog. Oxf., 70, 17 (1986).

27. D. C. Bassett, CRS Crit. Rev. Sol. St. Mat. Sci., 12, 97 (1984). 